\title{
A descontinuidade do campo elétrico em uma distribuição superficial e esférica de cargas: um invariante topológico
}

The discontinuity of the electric field in a spherical charge distribution: a topological invariant

\author{
Gustavo Elia Assad ${ }^{* 10}$ \\ ${ }^{1}$ Instituto Federal da Paraiba, João Pessoa, PB, Brasil.
}

Recebido em 03 de novembro de 2020. Aceito em 15 de dezembro de 2020.

\begin{abstract}
Este artigo soma-se aos outros recentes que vêm sendo publicados, principalmente nesta revista que está, ao nosso ver, tornando-se um fórum de discussão deste que é o campo elétrico na superfície de um condutor esférico carregado. Recentemente publicamos um artigo que discutia três modelos para encontrar o recalcitrante fator $1 / 2$ que persegue muitos aficionados por esta questão eletrostática. Desta forma, este texto foi elaborado, exclusivamente, para tentar esclarecer e contrapor, algumas interpretações colocadas em um mais recente artigo [1] que defende uma integral própria para encontrar o teimoso fator. Desta forma, reabordamos o método da superposição do campo de anéis para discutir comportamento do campo através de uma superfície esférica carregada uniformemente, tentando chegar a um consenso.
\end{abstract}

Palavras-chave: campo elétrico de anéis, esfera carregada, descontinuidade.

\begin{abstract}
This article adds to the other recent ones that have been published, mainly in this magazine that is, in our view, becoming a forum for discussion of this which is the electric field on the surface of a charged spherical conductor. We recently published an article that discussed three models for finding the recalcitrant factor $1 / 2$ that chase many fans of this electrostatic issue. In this way, this text was elaborated, exclusively, to try to clarify and oppose, some interpretations put in a later recent article [1] that defends a proper integral to find the stubborn factor. In this way, we reaffirm the method of superposition of the ring field to discuss the field's behavior through a uniformly charged spherical surface, trying to reach a consensus.
\end{abstract}

Keywords: electric field of rings, charged sphere, discontinuity.

\section{Introdução}

$\mathrm{Na}$ intenção de esclarecer o comportamento do campo elétrico sobre e através da superfície de um condutor esférico com distribuição superficial de cargas ( $\sigma$ é considerado constante em qualquer situação mostrada ao longo da discussão deste texto), trazemos, motivados por um artigo muito recente publicado nesta revista por Lima [1, um contraponto ao que esta referência tenta "demonstrar", refutando os argumentos por nós colocados, em [2. Na Ref. [1, o autor conclui que, para um modelo de distribuição superficial de cargas:

O campo elétrico é então descontínuo na superfície de uma esfera condutora, saltando de 0 para $\sigma /\left(2 \varepsilon_{0}\right)$ quando passamos de pontos dentro da esfera para qualquer ponto em sua superfície, e então deste para $\sigma / \varepsilon_{0}$ para pontos imediatamente externos a esfera.1

\footnotetext{
*Endereço de correspondência: gustavo.elia.assad@gmail.com

1 Texto traduzido para a língua portuguesa.
}

Indicando que o comportamento do valor campo elétrico através da superfície de um condutor é dado conforme a representação da Figura 11. Mostraremos, outra vez, que a regularização da integral imprópria encontrada, leva ao fator $1 / 2$ que representa o campo gerado por todas as cargas na vizinhança $\mathrm{P}$, excetuando-se

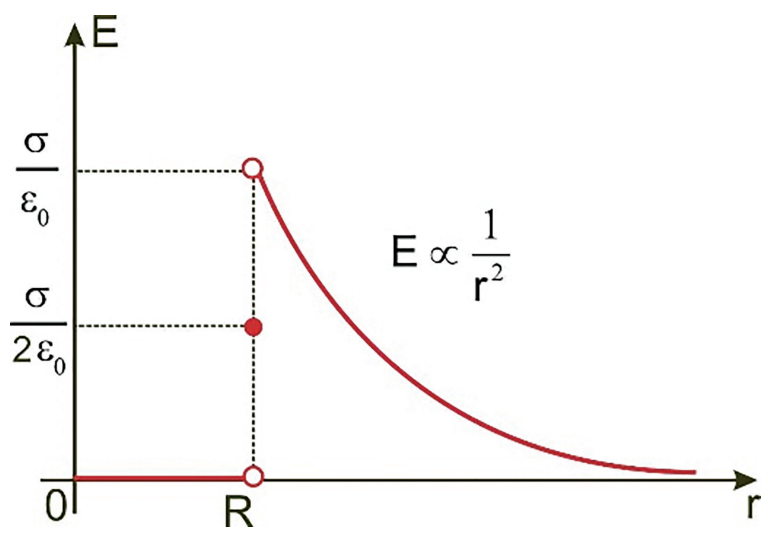

Figura 1: Comportamento gráfico do campo elétrico em uma distribuição esférica superficial de cargas, segundo a Ref. [1]. 
a carga do elemento de superfície que encerra P. Isto não indica, em absoluto, que o comportamento do campo se dá como o representado na Figura 1 .

\section{O Método da Superposição do Campo de Anéis}

Embora não achemos que este procedimento seja o mais simples para a determinação destes resultados (a lei de Gauss é rápida e suficiente para nos mostrar o que vemos na Eq. (9) e a descontinuidade na superfície. Lembremo-nos de Occam e sua navalha!), a superposição do campo de anéis tem sido usada para a discussão do campo elétrico na superfície em alguns artigos recentes publicados na RBEF, são eles [1-3] e [4]. Desta forma, para efeito comparativo direto, ipsis litteris por Lima [1], reproduziremos o ubíquo resultado do campo de um anel carregado com carga dQ, apoiado sobre uma esfera (Figura 2), gerando em um ponto da superfície um campo dado por:

$$
d E_{Z}=k_{0} \frac{(R-z)}{\left[r^{2}+(R-z)^{2}\right]^{\frac{3}{2}}} d Q
$$

Usando $z=R \cos \theta$ e $r=R \operatorname{sen} \theta$ e $d Q=\sigma(2 \pi r R d \theta) 2^{2}$ encontra-se:

$$
d E_{Z}=\frac{\pi}{\sqrt{2}} k_{0} \sigma \frac{\operatorname{sen} \theta}{\sqrt{1-\cos \theta}} d \theta
$$

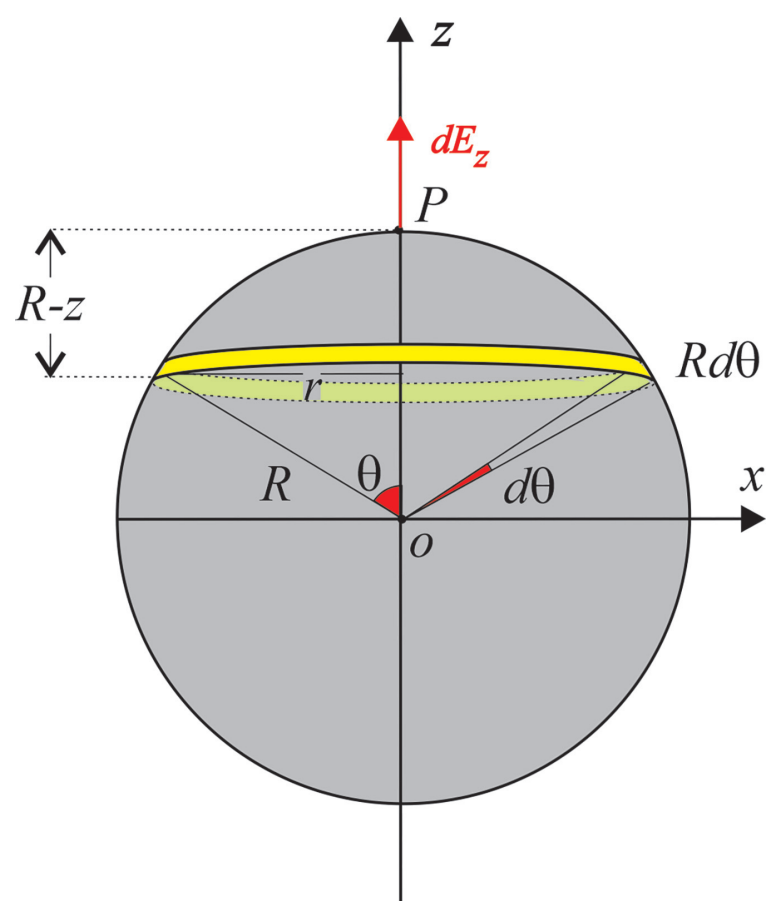

Figura 2: Representação do anel de carga dQ uma esfera de raio $\mathrm{R}$ e o campo que ele produz ao longo do eixo de simetria no ponto $P$

\footnotetext{
${ }^{2} \mathrm{O}$ elemento de carga pode ser escrito, sem perda de generalidade, por $d Q=2 \pi \sigma R^{2} \operatorname{sen} \theta d \theta$
}

$\mathrm{Ou}$

$$
d E_{Z}=\frac{k_{0} d Q}{2 R^{2} \sqrt{2(1-\cos \theta)}}
$$

Tomando as identidades $\operatorname{sen}(\theta)=2 \operatorname{sen}(\theta / 2) \cos (\theta / 2) \mathrm{e}$ $\operatorname{sen}^{2}(\theta / 2)=(1-\cos \theta) / 2$, tem-se:

$$
d E_{Z}=\pi k_{0} \sigma \cos \left(\frac{\theta}{2}\right) d \theta
$$

ou

$$
d E_{Z}=\frac{k_{0} d Q}{4 R^{2} \operatorname{sen}\left(\frac{\theta}{2}\right)}
$$

Agora, temos três grupos de equações para descrever o mesmo campo gerado por um anel carregado com carga $d Q$. Qual o campo $d E z$ para $r=0, z=R$ ou $\theta=0$ ? Nitidamente, as Equações (1) e (2) mostram que ele é indefinido neste ponto, o que o Lima também concorda [1]. Então, quando passamos para a Equação (3), onde deveríamos obter o mesmo resultado, Lima diz que, agora, o campo é definido neste ponto. A descontinuidade/continuidade é um invariante topológico e as três equações devem e mostram o mesmo resultado. Uma mudança de variável não faz a função (campo) deixar de ser descontínua num ponto $(z=R)$, e não fará $d E_{z}$ ser definido neste ponto. A reinclusão do ponto representativo de $z=R$ (ou $\theta=0$ ), feita pelo autor, mostra-se equivocada. Qualquer variação da Equação (1) ou integrações para a determinação do campo em $\mathrm{P}$, devem valer em $[-R, R)$ ou seu análogos, inclusive a Equação (3a).

Uma carga não gera campo nem força sobre ela mesma. Basta fazer $r=0$ e $z=R$ na Eq. (1); e $\theta=0$ nas Eqs. (2a 2b e (3b). Na Equação (3a), a indefinição do campo fica obscurecida pela manipulação algébrica proposta, mas é facilmente vista quando $d \theta$ é trocado por uma expressão com $d Q$ (i.e. $d Q=2 \pi \sigma R^{2} \operatorname{sen} \theta d \theta$ ), conforme mostrado na Eq. (3b).

Para ratificar a argumentação acima, observe a Figura 3 e apliquemos a lei de Gauss sobre o elemento

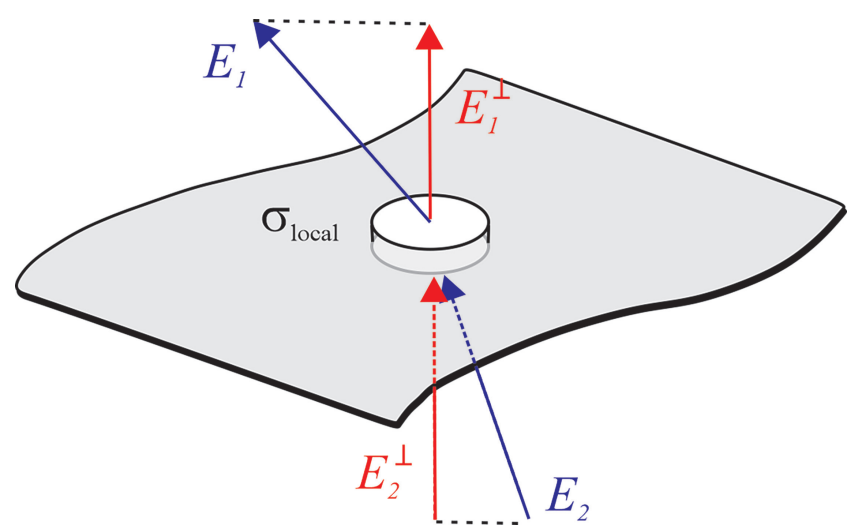

Figura 3: Descontinuidade da componente normal do campo elétrico através de uma superfície carregada. 
de superfície cilíndrico (pill box) para encontrar a conhecida equação da descontinuidade, aqui mostrada na Equação (4) encontrada na ampla literatura de nível superior (e.g. pág. 31 da Ref. [5]).

$$
E_{1}^{\perp}-E_{2}^{\perp}=\frac{\sigma_{\text {Local }}}{\varepsilon_{0}}=\frac{1}{\varepsilon_{0}} \frac{d Q_{\text {Local }}}{d A}
$$

A descontinuidade em uma superfície carregada é uma função da carga local, ou melhor, da densidade de carga local. Se não há carga em um elemento de área da superfície não há descontinuidade através deste elemento. Tomando o bem conhecido livro de eletrodinâmica de David Griffith 6, veja a transcrição de um fragmento de texto da pág. 102, que reitera os argumentos acima.

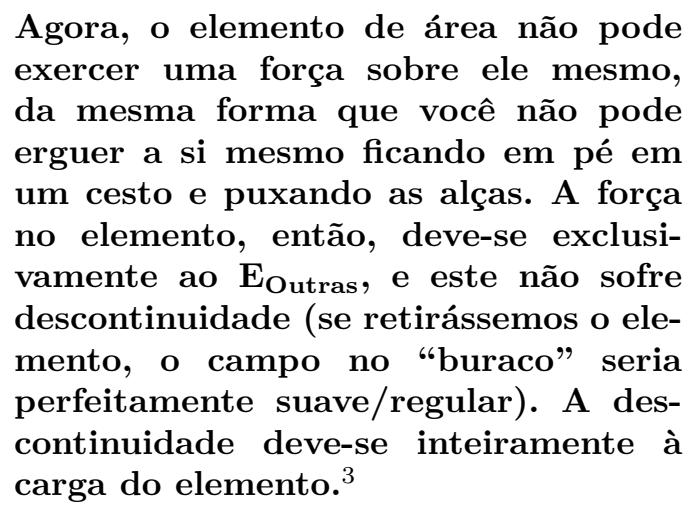

Mesmo diante da argumentação de Griffith [6], um resultado ubíquo na literatura, o autor (em [1) afirma que a presença de uma carga elétrica $(d Q)$ em um ponto não cria um campo infinito neste ponto e que o campo dela pode ser definido sobre ela mesma. Como argumento, usa o conhecido campo no interior de uma esfera uniformemente carregada (i.e. $\left.E(r)=\left(k|Q| / R^{3}\right) r\right)$. Este é o campo da carga líquida contida no interior de uma superfície gaussiana $\left(q_{\text {Gauss }}\right)$ de raio $r$, sobre esta superfície (i.e. $k\left|q_{\text {Gauss }}\right| / r^{2}$ ). Este é um excelente resultado que mostra que, conforme em [2], se há uma distribuição volumétrica de cargas, em casca ou esfera, existe uma transição contínua do campo através desse volume até a superfície. Este resultado não mostra o campo de um elemento infinitesimal desta distribuição sobre ele mesmo.

Em outro trecho, Lima afirma que a Eq. (1) não apresenta uma singularidade não integrável em $z=R$ (extensivo para as Eqs. (2) e (3)) sob o argumento de que o ponto $\mathrm{P}$ é encerrado por uma área nula e, portanto, não está associado a uma quantidade finita de carga, não podendo, assim, ser tratado como uma carga puntiforme. Se não houvesse carga em P:

(i) a densidade local de cargas seria nula e não haveria descontinuidade alguma;

(ii) se $\sigma=0$ e $d Q=0$, as Equações $2 \mathrm{a} \mid 2 \mathrm{~b}$ e $3 \mathrm{a} / 3 \mathrm{~b}$, obviamente, levariam ao valor $E_{P}=0$, mostrando

\footnotetext{
3 Texto traduzido para a língua portuguesa.
}

que este é um ponto "vazio" e esse elemento em nada influencia na superposição dos anéis. Em suma, o campo calculado em $\mathrm{P}$ só se deve ao restante das cargas, é dado por todas as cargas, menos a do anel de $\mathrm{P}$;

(iii) o campo evoluiria suavemente de dentro para fora (de 0 a 1 ou 0 a $\sigma / \varepsilon_{0}$ ), passando por infinitos valores e até mesmo por $1 / 2$ na superfície, mas, de forma alguma, seria descontínuo ou representado pelo gráfico da Figura 1, defendido pelo Teorema encontrado em [1]. (Vide transcrição do Griffith acima);

(iv) não haveria força radial para fora sobre o elemento de superfície em $\mathrm{P}$ nem sobre qualquer outro elemento da superfície da esfera ${ }^{4}$

(v) não haveria pressão eletrostática alguma sobre a superfície da esfera. ${ }^{5}$

Sendo assim, existe sim um elemento de carga não nulo em ambos os anéis diametralmente opostos da esfera (em $z= \pm R$ ) dados, justamente, por $d Q=\sigma d A$ (para que $\sigma$ seja constante, finito e diferente de zero, $d Q$ e $d A$ são não nulos). Contudo, para mostrar que este elemento de carga se comporta como uma carga puntiforme, façamos $r=0$ e $d=R-z$ na Eq. (1), encontrando a expressão para o campo de uma carga puntiforme a uma distância " $d$ " não nula da mesma e que não está definido em $d=0$ (ou $z=R$ ).

$$
d E_{Z}=\frac{k_{0} d Q}{d^{2}}
$$

Em um caso particular de um ponto sobre a esfera, podemos fazer $z=-R$ na Eq. (1); e $\theta=\pi$ nas Eqs. (2b) e $(3 \mathrm{~b})$, encontrando, em todas,

$$
d E_{Z}=\frac{k_{0} d Q}{4 R^{2}}
$$

Esta é a expressão do campo de uma carga puntiforme $d Q$ a uma distância $2 R$ da mesma. Obviamente, isto ocorre pois $d=2 R>>R d \theta$. Ao nos aproximarmos muito do elemento de carga $d Q$, que ocupa a superfície $d A$ em $z=R$, ele irá se comportar como um pequeno disco carregado, gerando em pontos próximos à sua face um campo de módulo $\frac{\sigma}{2 \varepsilon_{0}}$ para dentro e para fora da esfera. Não estamos dizendo que o campo do disco é infinito, estamos dizendo que este pequeno disco gera campos com valores finitos e sentidos opostos em suas faces $\left( \pm \frac{\sigma}{2 \varepsilon_{0}}\right)$ e que o campo não está definido sobre a superfície, sobre o próprio disco, onde $d=0$ ou $z=R$. Então, o restante das cargas deve gerar um

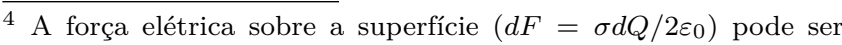
vista na página 30 da Ref. [7.

5 Se tomarmos a expressão da nota de rodapé anterior e substituirmos dQ por $\sigma \mathrm{dA}$, encontramos a força por unidade de área ou a pressão eletrostática $\left(d F / d A=p=\sigma_{2} / 2 \varepsilon_{0}\right)$ que pode ser vista na Ref. 6] ou na página 32 da Ref. 7], inclusive com um cálculo de um valor típico de $3,6.10^{-4} \mathrm{~N} / \mathrm{m}^{2}$.
} 
campo em $\mathrm{P}\left(+\frac{\sigma}{2 \varepsilon_{0}}\right)$ de dentro para fora (é aí que está o recalcitrante fator $1 / 2$ ), de modo a fazer com que o campo interno seja nulo e o externo seja $\frac{\sigma}{\varepsilon_{0}}$. Mais adiante, como já feito em [2, mostraremos como se comporta o campo quando "viajamos" através de um orifício na esfera, tal qual através do ponto $\mathrm{P}$ quando se retira a carga $d Q$. Passaremos por $1 / 2$ e não saltaremos para ele.

\section{A Integração}

Admitindo-se, como dito por Jackson [5, Griffith 6], Purcell [7, etc., que o campo é descontínuo na superfície de um condutor com distribuição superficial de cargas, no caso da geometria esférica e com o método da superposição de anéis, a superposição de todos os anéis sobre a esfera gera uma singularidade não integrável em pontos da superfície. Assim, para regularizar a integral, exclui-se o ponto do domínio em que há descontinuidade (em que o integrando diverge) e procede-se com o limite de aproximação de P. Método já mostrado e bem fundamentado em [2, 3] e [4]. Para generalizar, considere $d E_{z}$ função de uma variável qualquer (e.g. $d E_{z}=f(w)$ ), com descontinuidade em $w=a(f(a)$ é indefinido). O procedimento bem conhecido de regularização da integral impropria é:

$$
\int_{a}^{b} f(w) d w=\lim _{k \rightarrow a} \int_{k}^{b} f(w) d w
$$

Desta forma, os procedimentos de integração associados às Eqs. (1 3), retirando-se um elemento infinitesimal de carga $(d q)$ da superfície, levariam ao mesmo fator $1 / 2$. Isto é, o campo gerado por todas as cargas da vizinhança de $\mathrm{P}\left(E_{Q-d q}\right)$, excluindo-se a carga deste elemento de superfície seria radial para fora e dado por:

$$
\left.E_{Q-d q}\right|_{z=R}=\frac{1}{2} k_{0} \frac{Q}{R^{2}}=\frac{\sigma}{2 \varepsilon_{0}}
$$

Onde $Q$ é a carga total da esfera. Este campo, ao se superpor ao campo do elemento de superfície em $z=R$, zera o campo no interior, faz o campo ser $\frac{\sigma}{\varepsilon_{0}}$ em pontos externos muito próximos da esfera e cria descontinuidade em $z=R$.

Com todo o rigor matemático, na Ref. [2] é mostrada uma expressão para o cálculo do campo de "todos" os anéis em um ponto qualquer do diâmetro da esfera e não só na superfície. A equação na forma dimensional é mostrada a seguir na Equação (8). O eixo de simetria foi tomado no eixo z para efeito comparativo com [1].

$$
E(z)=\frac{\sigma}{2 \varepsilon_{0}} \frac{R}{z}\left[\frac{-R+z}{\sqrt{(-R+z)^{2}}}+1\right], \quad z \neq \pm R
$$

A descontinuidade no valor do campo pode ser rigorosamente obtida quando se faz o limite de $E(z)$ com $z$ tendendo a $\mathrm{R}$ pela direita e pela esquerda, além de que, $E(R)$ é indefinido, a função $E(z)$, como esperado, apresenta uma descontinuidade na superfície, conforme mostra a Figura 4. Na Figura 5, coloca-se o conhecido gráfico do potencial elétrico com destaque para a não diferenciação do mesmo com respeito a $\mathrm{z}$, em $z=R$, ratificando a descontinuidade do campo.

$$
\left\{\begin{array}{l}
\lim _{z \rightarrow R^{-}} E(z)=0 \\
\lim _{z \rightarrow R^{+}} E(z)=\frac{\sigma}{\varepsilon_{0}}
\end{array}\right.
$$

Agora, se retirarmos o elemento de carga do elemento de área onde se encontra $\mathrm{P}$, conforme as palavras citadas de David Griffith 6], o campo deve evoluir suavemente entre seus notórios valores de " 0 " no interior da esfera até " $\frac{\sigma}{\varepsilon_{0}}$ " em pontos justapostos à sua superfície. Para verificar este comportamento, em [2, o procedimento de retirada do elemento de carga é feito através da alteração do limite de integração, tal qual feito nas demais referências associadas, levando ao seguinte resultado:

$$
E(z, \xi)=\frac{\sigma}{2 \varepsilon_{0}} \frac{R}{z}\left(1+\frac{-R+z \cos (\xi)}{\sqrt{R^{2}+z^{2}-2 R z \cos (\xi)}}\right)
$$

Esta equação mostra que quando $\xi \rightarrow 0$, o campo neste pequeno orifício é " $\frac{\sigma}{2 \varepsilon_{0}}$ " e que não há, de fato, descontinuidade alguma entre pontos internos e externos. Os limites laterais são absolutamente iguais e também assumem este valor. Vide Eq. (11).

$$
\lim _{\xi \rightarrow 0} E\left(z \rightarrow R^{+,-}, \xi\right)=\frac{\sigma}{2 \varepsilon_{0}}
$$

Para mostrar a transição do campo através do orifício e a confirmação da não descontinuidade, observe a Figura 6 que mostra a plotagem de três gráficos representativo da Eq. $(10)$, com $\xi=10^{-7} \mathrm{rad}$ em três escalas diferentes.

Estes gráficos, com escalas horizontais diferentes, mostram a evolução do campo quando "viajamos" através do

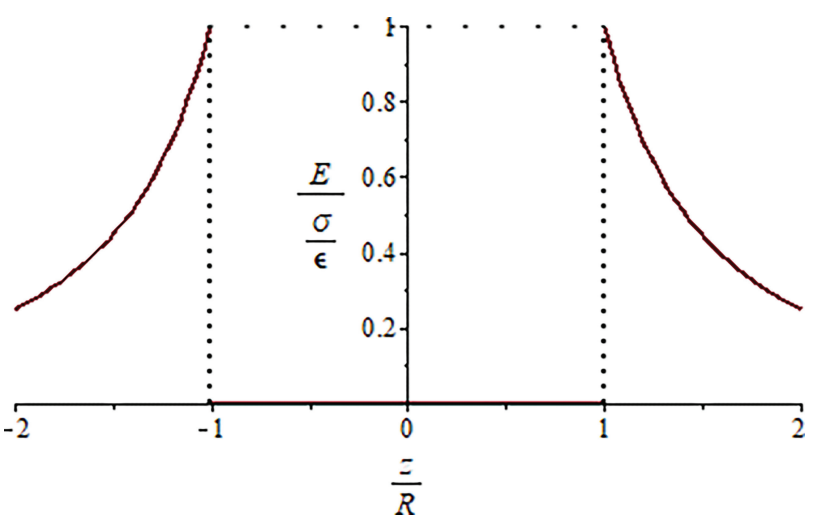

Figura 4: Descontinuidade do campo elétrico na superfície do condutor esférico. 


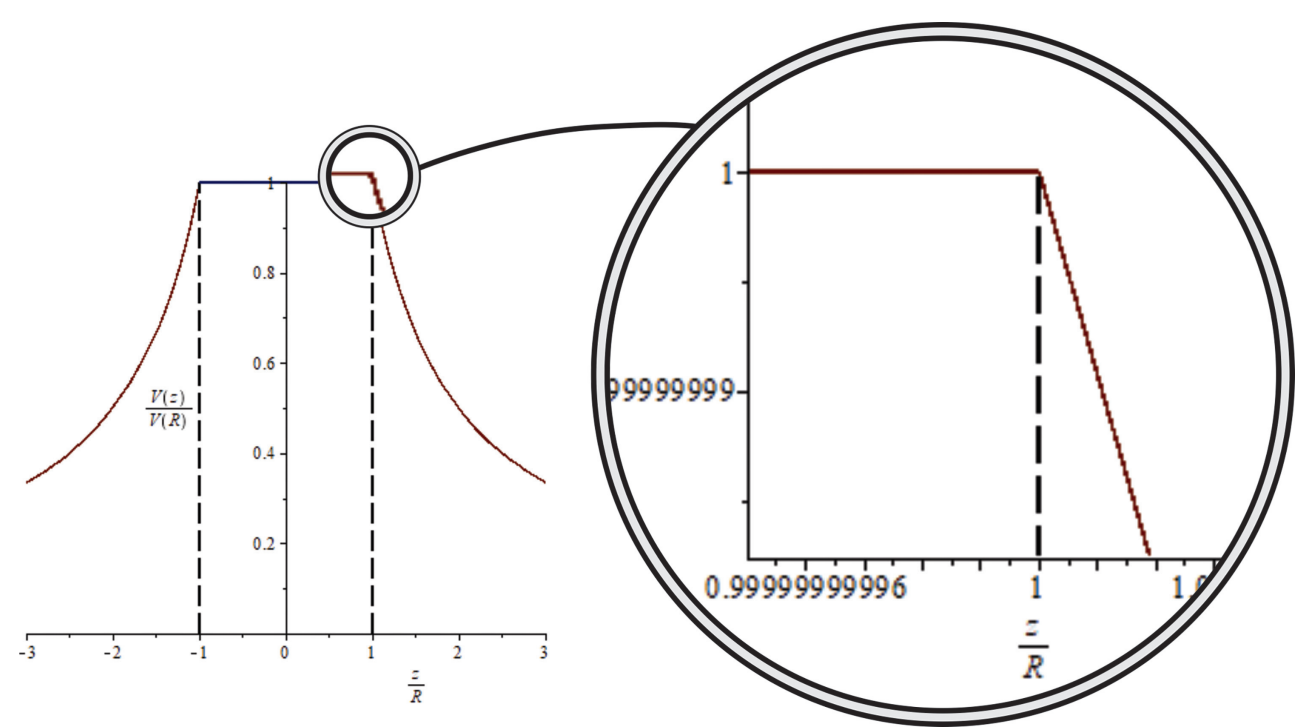

Figura 5: Gráfico de $\mathrm{V}(\mathrm{z})$ com destaque para o fato de que esta função é NÃO derivável em $z=R$ (superfície).
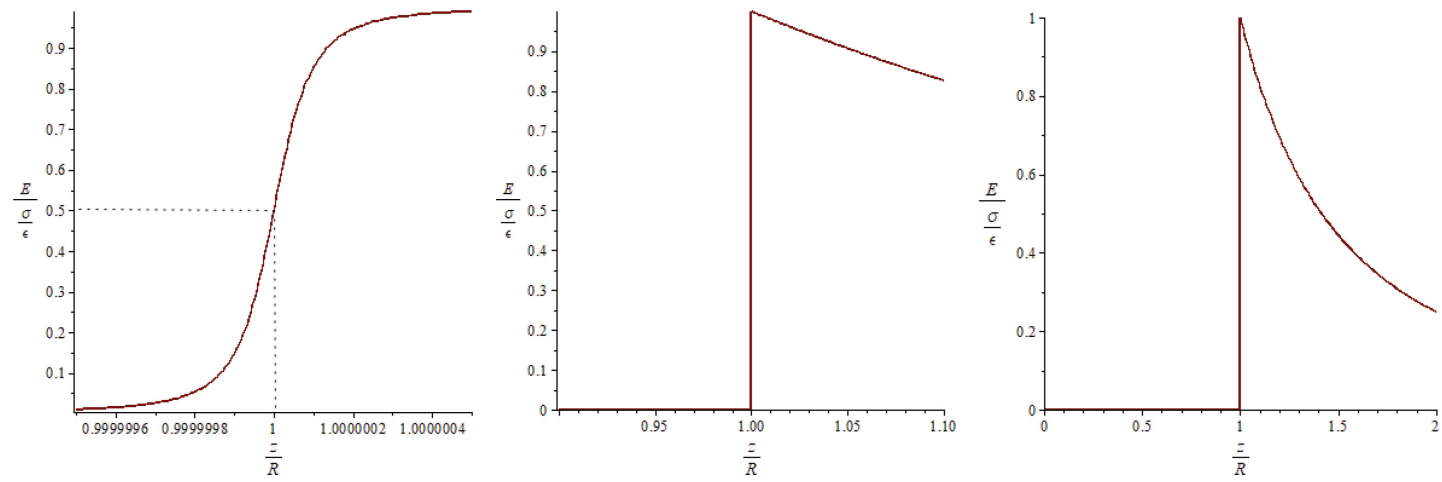

Figura 6: Gráficos que mostram a evolução do campo através do elemento de área carregado retirado da superfície do condutor no ponto $P$.

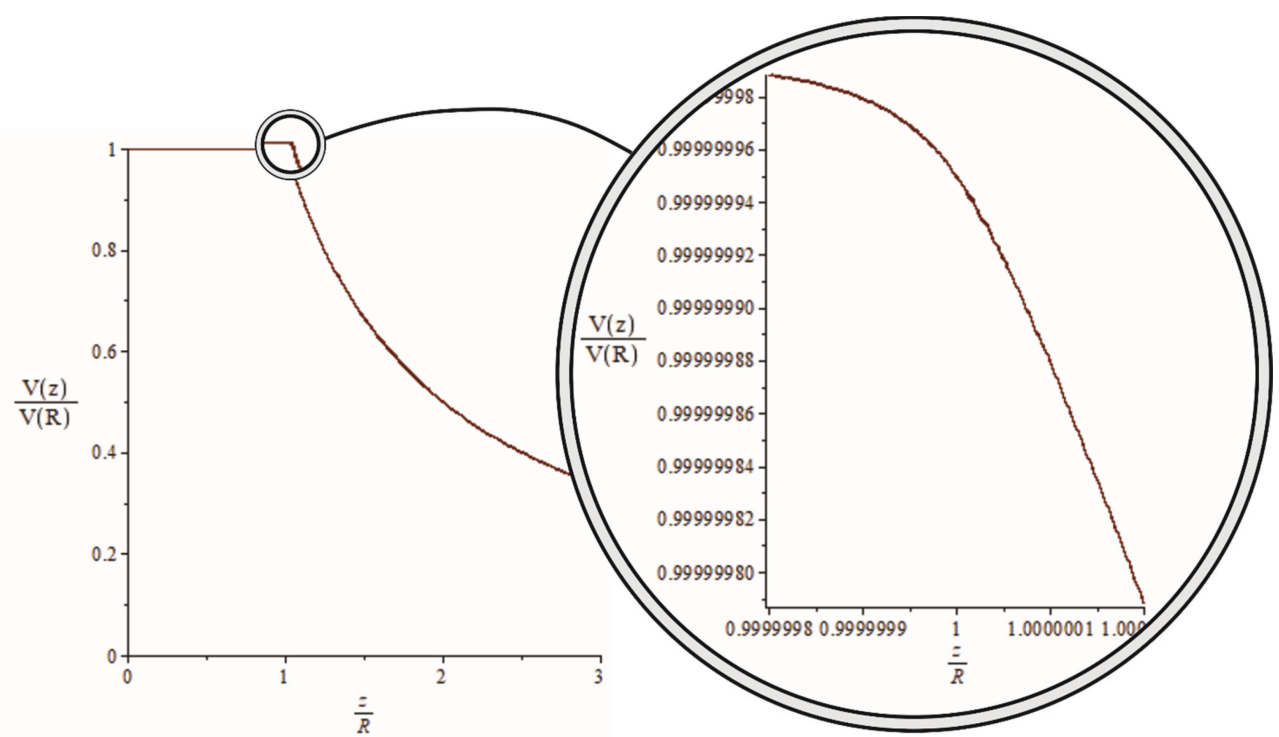

Figura 7: Comportamento do potencial elétrico em função da posição, ao longo de uma direção radial com destaque para a transição entre pontos internos e externos próximos do "buraco" deixado pela retirada do elemento de carga dq. 
orifício de onde foi retirada a carga da superfície para eliminar a descontinuidade do campo. Ao ponto que nos afastamos da esfera, vemos uma transição súbita do valor do campo e, quando próximos, vemos que, de fato, o campo evolui suavemente através desse, mostrando mais uma vez que, em se retirando o elemento de superfície, a descontinuidade não mais existe. Não obstante, como o campo é contínuo em todo o domínio e sabendo que $E(z)=-\frac{\partial V}{\partial z}$ mostra-se, na Figura 7 o comportamento do potencial elétrico ao longo do eixo $z$, com destaque para pontos muito próximos de $z=R$, de onde foi retirado o elemento de carga e por onde transita-se através do "buraco". Este destaque mostra que $V(z)$ está longe de estar associado à transição do campo sugerida em [1] e representada no gráfico da Fig. 1] Sugere-se, enfim, que o leitor se indague: como seria o comportamento do potencial se a transição do campo fosse, de fato, como mostrada na Fig. 1?

\section{Conclusões}

Após análise da metodologia adotada por [1], observamos que o uso da superposição de anéis para a determinação do campo elétrico em um ponto da superfície carregada de um condutor esférico apresenta um nítida descontinuidade em $z=R$, evidenciada, exatamente, a partir da fórmula clássica do campo elétrico gerado por um anel em pontos do eixo de simetria do mesmo, Eq. (1). O campo não deixa de ser descontínuo neste ponto $(z=R$ ou $\theta=0$ ), nem a função não passa a ser integrável nele, por uma mudança de variável. A descontinuidade tem invariância topológica. Todas as Equações advindas de (1) devem ser aplicáveis em todos os pontos do domínio, excetuando-se $z=R$, inclusive a Equação (3a), através da qual Lima 1 afirma que o ponto pode ser reincluído e que leva ao suposto salto de $E=0$ até $E=\frac{\sigma}{2 \varepsilon_{0}}$ e deste para $E=\frac{\sigma}{\varepsilon_{0}}$. Sendo assim os limites de integração associados às Equações (1-3) devem ser $[-R, R)$ e $(0, \pi]$. Ainda, conforme o próprio autor, a retirada deste ponto do domínio não afeta o valor da integração e leva ao mesmo fator $1 / 2$ (associado ao Valor Principal de Cauchy para integrais impróprias) mas, não faz o campo variar de acordo com a Fig. 1 O campo transitaria suavemente de $E=0$ a $E=\frac{\sigma}{\varepsilon_{0}}$ através do orifício deixado em $\mathrm{P}$, conforme dito por $[6] \mathrm{e}$ demonstrado por [2] com a Eq. (10) e a representação gráfica na Fig. 5. Isto não é nada além de estarmos dizendo que a descontinuidade do campo elétrico em uma superfície carregada é dada, exclusivamente pela carga presente no elemento de superfície, pela densidade de cargas local. Excluindo-se este elemento, não há mais descontinuidade, conforme ratificado pela famosa Ref. [5].
Por fim, se o modelo adotado é o de uma distribuição superficial esférica de cargas, o gráfico representativo é o da Figura 4 e sugere-se:

"Para qualquer esfera condutora de raio $R$ e densidade superficial $\sigma$, em equilíbrio eletrostático, o campo elétrico é descontinuo em pontos de sua superfície, nulo em seu interior e $\frac{\sigma}{\varepsilon_{0}}$ para pontos da superfície externa quando, então, cai com a lei do inverso do quadrado da distância ao centro da esfera."

\section{REFERÊNCIAS}

[1] F.M.S. Lima, Rev. Bras. Ensino Fís. 42, e20200182 (2020)

[2] G.E. Assad, Rev. Bras. Ens. Fís. 42, e20190245 (2020)

[3] K. Slodkowski, M.C. Pinheiro e T.C. Luchese, Rev. Bras. Ensino Fís. 40, e2311 (2018).

[4] F.M.S. Lima, Resonance 23, 1215 (2018)

[5] J.D. Jackson, Classical Electrodynamics: (Wiley, NewYork, 1998), 3rd ed., p. 31.

[6] D.J. Griffiths, Introduction to Electrodynamics (Prentice Hall, Upper Saddle River-NJ, 1999), p. 102.

[7] E.M. Purcell e D. J. Morin, Electricity and Magnetism: 3rd ed. (Cambridge University Press, New York, 2013), v. 2 . 